\title{
La rifaximina fue eficaz y segura para el tratamiento del síndrome de intestino irritable
}

Rifaximin was safe and efficacious for irritable bowel syndrome

Menees SB y col. The Am J Gatroenterology 2012.107:28-38.

\section{Objetivos}

Estimar la eficacia y seguridad del tratamiento con rifaximina para el síndrome de intestino irritable (SII).

\section{Fuentes de datos, selección de estudios y extracción de datos}

Los autores realizaron una búsqueda sistemática en Medline, Embase, Cochrane Library y Web of Science incluyendo todos los ensayos clínicos aleatorizados doble ciegos que compararan rifaximina vs. placebo en pacientes con SII, definido por los criterios de Manning, Roma I, Roma II o Roma III, sin restricción de lenguaje, búsqueda manual en tres revistas relevantes de gastroenterología y búsqueda de artículos referenciados. Dos investigadores independientes seleccionaron y extractaron los estudios y un tercero evaluó las discrepancias. Para evaluar la calidad de cada estudio se utilizo la herramienta de valoración de riesgo de sesgos de la Colaboración Cochrane.

\section{Resultados Principales}

De las 13.700 citas halladas solamente cinco cumplieron con los criterios de elegibilidad. El meta-análisis reveló que la rifaximina fue más eficaz que placebo en la mejoría global de los síntomas del SII (desenlace primario) con un odds ratio $(\mathrm{OR})^{\star}$ de 1,57 (IC $95 \% 1,22$ a 2,01) y un número necesario a tratar
$(\mathrm{NNT})^{*}$ de 10,2 durante 10 a 14 días de tratamiento, mostrando a su vez una heterogeneidad leve (I2 26\%)*. Para el desenlace secundario, distensión abdominal, se obtuvo información de cuatro estudios revelando que la rifaximina fue mejor que el placebo con un OR de 1,55 (IC 95\% 1,23 a 1,96) con un NNT de 10,1 durante 10 a 14 días de tratamiento sin heterogeneidad significativa ( $\left({ }^{2} 23 \%\right)$. En análisis de meta regresión*, se encontró que estudios con pacientes de mayor edad y mayor porcentaje de mujeres presentaron una mayor tasa de respuesta independientemente del tratamiento. Además se encontró que a mayor dosis acumulada mayor tasa de respuesta en todos los tratamientos. De las variables analizadas, la edad fue el factor de mayor predicción para la respuesta con un índice de correlación* de 0,97. Con respecto a los efectos adversos fueron similares en ambos grupos, y los más frecuentes $(<6 \%)$ con rifaximina fueron cefalea, infección de vías aéreas superiores, nauseas, diarrea y dolor abdominal.

\section{Conclusiones}

En el análisis efectuado la rifaximina demostró ser más efectiva que el placebo para la mejoría de los síntomas de SII en forma global y en distensión abdominal. La modesta ganancia terapéutica fue similar a las alcanzadas por otros tratamientos para SII y los efectos adversos fueron similares al placebo.

\section{Comentario}

En la actualidad el tratamiento del SIl es empírico, ponderando la severidad de los síntomas y orientado a paliar el síntoma predominante, ya sea diarrea, constipación o dolor abdominal ${ }^{1}$. En este aspecto la rifaximina suma una herramienta terapéutica más al grupo y si bien no se conoce con certeza el mecanismo de acción, las hipótesis planteadas y la buena evidencia encontrada, aunque escasa, hacen de este antibiótico un medicamento plausible. Si pensamos concretamente en nuestra práctica médica diaria estaremos de acuerdo en decir que los pacientes que presentan este cuadro resultan algunas veces difíciles de tratar, habiendo probado ya distintos tratamientos sin buena respuesta. Es por ello que la rifaximina se nos presenta como una opción más a nuestro arsenal terapéutico. Resulta cuestionable en cuanto a la valoración de los efectos adversos que no se haya tenido en cuenta un seguimiento más largo de los pacientes incluidos para evaluar la recurrencia de los síntomas y la tolerabilidad a largo plazo, sin embargo esto representa una limitación relacionada mas a los estudios originales y no a la revisión sistemática en si misma.

\section{Conclusiones del comentador}

La rifaximina es una herramienta más en el manejo de pacientes con SII que parece similarmente efectiva a las existentes actualmente. Es importante evaluar su efectividad y seguridad a largo plazo para que su adopción sea recomendada en forma rutinaria en pacientes con este frecuente problema de salud.

Agustín M. Segalini [ Servicio de Medicina Familiar y Comunitaria del Hospital Italiano de Buenos Aires. agustin.segalini@ hospitalitaliano.org.ar ]

Segalini A. La rifaximina fue eficaz y segura para el tratamiento del Síndrome de Intestino Irritable.Evid Act Práct Ambul. Jul-Set 2012;15(3):93. Comentado de: Menees SB, Maneerattannaporn M, Kim HM y col. The efficacy and safety of rifaximin for the irritable bowel syndrome: a systematic review and meta-analysis. Am J Gastroenterol. 2012 Jan107:28-38. PMID: 22045120.

\section{Referencias bibliográficas}

1. Kopitowski K. Colon irritable y constipación. En: Problemas digestivos. Rubinstein R, Zárate M, Carrete P, Deprati M, editores, 3ra Ed. Buenos Aires: Fundación MF "Para el desarrollo de la medicina familiar y la atención primaria de la salud", 2006. 